\title{
Staphylococcus aureus Bacteremia: What is the Impact of Oxacillin Resistance on Mortality?
}

\author{
Cassettari V.C. ${ }^{1}$, Strabelli T. $^{2}$ and Medeiros E.A.S. ${ }^{1}$
}

\author{
${ }^{I}$ Division of Infectious Diseases, Clinical Hospital of Medicine \\ Faculty of São Paulo University; ${ }^{2} \mathrm{Hospital}$ Infection Control \\ Group, Heart Institute of Medicine Faculty of São Paulo \\ University, São Paulo, SP, Brazil
}

\begin{abstract}
In order to analyse the impact of oxacillin resistance on the mortality of Staphylococcus aureus bacteremia, and to assess the antimicrobial susceptibility of community-acquired strains in two large university hospitals (the Instituto do Coração do Hospital das Clínicas da Faculdade de Medicina da Universidade de São Paulo and the Instituto Central do Hospital das Clínicas da Faculdade de Medicina da Universidade de São Paulo), we carried out a four-month-long prospective cohort study, which included 163 consecutive cases of $S$. aureus bacteremia. Of these, $140(85.9 \%)$ were hospital-acquired, $9(5.5 \%)$ were community-acquired and $14(8.6 \%)$ were of indeterminate origin. No cases of community-acquired infection by oxacillin-resistant $S$. aureus was identified. Among hospital-acquired infections, oxacillin-resistant $S$. aureus was responsible for $64.3 \%$ of cases. Mortality up to 15 days after diagnosis of bacteremia was $27 \%(18 / 67)$ for infections caused by susceptible strains and 33\% (32/96) for infections caused by oxacillin-resistant strains $(p=0.10)$. The following independent risk factors for the acquisition of oxacillin-resistant $S$. aureus were identified in multiple logistical regression analysis: age over 60 years, use of corticoids; presence of a central vascular catheter, and previous use of antibiotics.

Key Words: Staphylococcus aureus, bacteremia, mortality.
\end{abstract}

Concern about the treatment of S. aureus infections dates from the appearance of penicillin-resistant strains as early as the 1940's. Since then, penicillinase-resistant antibiotics - oxacillin and methicillin - have become available, but resistance to these antibiotics soon became apparent and has now reached high endemic levels throughout the world, especially in large hospitals. In the Instituto Central do Hospital das Clínicas da Faculdade de Medicina da USP (IC-HCFMUSP) the rate of resistance to oxacillin among hospital-acquired infections was 72\% during the first six months of 1999. Once established in a hospital, oxacillin-resistant $S$. aureus is extremely difficult to eradicate. The reasons

Received on 17 June 2004; revised 22 October 2004.

Address for correspondence: Dr. Valéria Cassettari Chiaratto. Rua Nazaré Paulista, 163 ap 123-Bl. Estoril. Zip code: 05448000, São Paulo-SP, Brazil. E-mail: valeriac@ hu.usp.br

The Brazilian Journal of Infectious Diseases 2005;9(1):70-76 (C) 2005 by The Brazilian Journal of Infectious Diseases and Contexto Publishing. All rights reserved. for this persistence have not been fully established, but the importance of person-to-person transmission, with the patients themselves as the source and the professional hospital staff as the carriers, has already been clearly shown.

As an agent of community-acquired infection, oxacillin-resistant $S$. aureus is becoming increasingly important and has been the subject of a number of studies [2-4]. A recent study carried out in the Hospital São Paulo showed that $4.4 \%$ of the cases of oxacillinresistant $S$. aureus bacteremias seen there from 199192 were community-acquired, whereas the percentage was $15.9 \%$ for $1995-96$, indicating an increasing trend in our community [5].

The first case of vancomycin intermediate-resistant S. aureus infection was identified in Japan in 1996, and since then various cases have been described in other countries, including Brazil. The risk factors identified were prior use of vancomycin and previous oxacillin-resistant S. aureus infection [6-9]. Studies of 
the strains showed that there had been no acquisition of $v a n A$ or $v a n B$ genes, which confer vancomycin resistance in enterococci, except for two cases of isolation of $S$. aureus that were both vancomycinresistant and carriers of the VanA gene [10].

The importance of determining the rate of occurrence and the factors predisposing to community-acquired oxacillin-resistant $S$. aureus arises from the need to standardize the initial treatment of suspected cases. Rigorous criteria should always be used when recommending vancomycin, as it exerts selective pressure on, and induces resistance in, enterococci and possibly $S$. aureus itself. There is also evidence that it is less effective than oxacillin [11].

With regard to the prognosis and mortality associated with $S$. aureus bacteremia, there is no consensus as to the impact of oxacillin resistance on the evolution of these infections. Studies have given discordant results $[4,12,13]$, although a recently published meta-analysis showed an association between resistance and greater mortality [14].

In our setting, the negative impact of oxacillinresistant $S$. aureus bacteremia on mortality and hospitalization time has already been clearly shown [15]. In comparison with the evolution of oxacillinsusceptible $S$. aureus bacteremias, a previously cited study [5] observed greater mortality for S. aureusresistant bacteremias, while raising the possibility that this may have been due to the greater virulence of the strain detected as dominant in the hospital where it was isolated.

The main aims of our study were to analyze the impact of oxacillin resistance on the mortality of $S$. aureus bacteremias and to assess the antimicrobial susceptibility of domestically-acquired strains in our setting. A secondary aim of the study was to identify variables associated with oxacillin resistance in the study population.

\section{Material and Methods}

A four-month-long prospective cohort study was made from $5 / 1 / 99$ to $8 / 30 / 99$. The study included cases of $S$. aureus bacteremia seen at the Instituto Central do Hospital das Clínicas da Faculdade de Medicina da USP (IC-HC) and the Instituto do Coração do Hospital das Clínicas da Faculdade de Medicina da USP (InCor), which are part of the hospital complex associated with the Faculdade de Medicina da USP.

The IC-HC is a primarily public tertiary university hospital, with some beds reserved for health plan patient care. There are a total of 875 beds, of which 90 are in the Intensive Care Unit; 21,400 surgical operations were carried out in 1998. It has a neonatal Intensive Care Unit and a burns department, and liver, kidney and bone marrow transplants are carried out, as well as other high-complexity procedures.

InCor is a tertiary university hospital providing public and health plan patient care, which specializes in cardiac diseases and carries out high complexity procedures in this field. It has 377 beds, of which 90 are in the Intensive Care Unit. It carried out 2,440 surgeries in 1998, including heart transplants.

Cases of S. aureus positive blood cultures collected sequentially over the three-month period were obtained from the microbiology laboratory. Each patient was only included once in the study. Information on each patient was obtained by means of clinical follow-up, up to 48 hours after the result of each blood culture.

Blood cultures were processed in accordance with NCCLS - National Committee for Clinical Laboratory Standards [16], with cultures grown in Bactec Plus bottles and antibiograms carried out using the agar gel diffusion method. Classification as hospital-acquired, community-acquired or of indeterminate origin was based on the following criteria:

1) Hospital-acquired: blood cultures collected after at least 48 hours of hospitalization, or from a patient transferred from another hospital, or from a patient coming from home who had been hospitalized for less than 48 hours, but who had been hospitalized and had a surgical procedure carried out within the last month, or within the last year in the case of a prosthetic implant.

2) Community-acquired: blood cultures collected within 48 hours of hospitalization from patients who were not 
being followed in a day hospital and who had not been hospitalized during the previous year.

3) Indeterminate origin: blood cultures collected within 48 hours of hospitalization from patients who were being followed in a day hospital or had been hospitalized in the last year but without undergoing surgery during this period.

The primary focus of infection was defined in accordance with the criteria established by the NNISS - National Nosocomial Infectious Surveillance System [17]. In the absence of another focus, bacteremic central catheter-related infection was defined to be the presence of a positive blood culture and either a quantitative culture of the catheter tip with more than $15 \mathrm{CFU}$, or the presence of signs of inflammation in the insertion area.

We recorded the following risk or comorbidity factors for each patient: diabetes mellitus, cardiac insufficiency, chronic renal insufficiency, chronic obstructive pulmonary disease, chronic hepatic insufficiency, immunodeficiency associated with the human immunodeficiency virus $\left(\mathrm{CD}_{4} \leq 200\right)$, use of corticoids for more than one week, neoplasia, neutropenia ( $\leq 1,000$ neutrophils), burns, surgery during the two months preceding blood culture, chemotherapy, invasive mechanical ventilation, central catheter, indwelling bladder probe, pacemaker.

The antibiotics used in the month preceding blood culture were listed. We considered the total number of days of previous antibiotic therapy as the sum of the number of days of use for each antibiotic.

The evolution of each patient was assessed according to the type of discharge from the hospital and was classified into two categories: hospital discharge and death. Death was considered to be attributable to bacteremia when it occurred up to 15 days after blood culture.

The data were stored and analyzed using version 6 of the Epi-Info program. Multiple regression analysis was carried out using version 11 of the SPSS program.

\section{Results}

Characterization of the study population

One hundred and seventy-one patients with positive blood cultures were assessed. Eight of these were excluded because of lack of data; 163 patients included in the study. Distribution by sex was $59 \%$ male and $41 \%$ female. The average age was 50 years, ranging from 0 to 91 years; $79.2 \%$ (129/163) of the patients were seen in the IC-HC and 20.8\% (34/163) were seen at InCor. As regards the hospital wards, $6.1 \%$ $(10 / 163)$ of the cases came from the nursery, $1.8 \%$ (3/ 163 ) from outpatient departments, $14.7 \%$ (24/163) from emergency rooms, $41.7 \%$ (68/163) from clinical or surgical wards and $35.6 \%$ (58/163) from Intensive Care.

As regards the source of the infection, $85.9 \%$ (140/ 163 ) of the cases were classified as hospital-acquired, $5.5 \%$ (9/163) were community-acquired and 8.6\% (14/163) were considered of indeterminate origin. Among the latter, 57.1\% (8/14) had been hospitalized during the last month and $28.6 \%$ (4/14) in the last year, without undergoing surgery, and $14.3 \%(2 / 14)$ were followed in a day hospital. Of those classified as hospital-acquired, $92.9 \%$ (130/140) had blood cultures collected at least 48 hours after admission, 5.7\% (8/ 140) had been hospitalized in the last month and undergone surgery, $0.7 \%$ (1/140) had been hospitalized in the last year and undergone surgery with a prosthetic implant, and $0.7 \%(1 / 140)$ had been transferred from another hospital.

As regards the risk and comorbidity factors, the high prevalence of invasive procedures (central catheter $50.3 \%$; bladder probe $32.5 \%$ ), and previous surgery $(35.6 \%$ or $58 / 163)$ and use of corticoids $(22.1 \%$ or 36/163) were noticeable.

In order of frequency, the foci detected were: bacteremia without a known focus $25.2 \%$ (41/163), bacteremic central catheter-related infection $20.2 \%$ (33/163), pneumonia $15.9 \%$ (26/163), surgical site $12.3 \%$ (20/163), skin 6.1\% (10/163), endocarditis $4.3 \%$ (7/163), phlebitis $4.3 \%$ (7/163) and others $11.7 \%$ (19/163). 
As regards treatment, $52.1 \%(85 / 163)$ received a suitable antibiotic up to 24 hours after the blood culture was collected, 39.3\% (64/163) received a suitable antibiotic between 24 and 72 hours after the blood culture was collected and $8.6 \%$ (14/163) did not receive suitable antibiotics, or received them too late, more than 72 hours after the blood culture was collected.

Death occurred in $42.9 \%$ (70/163) of the total number of cases; $71.4 \%$ (50/70) of the deaths occurred up to 15 days after the date of the blood culture, and they are therefore, according to our criteria, attributable to the bacteremia. In hospital-acquired infections, death occurred in $42.8 \%$ (60/140) of the cases; in community-acquired infections it occurred in 33\% (3/9) and in those of indeterminate origin 50\% (7/14). As regards the foci, although a greater incidence of death was observed among cases of endocarditis ( $71 \%$ or $5 / 7)$ and pneumonia $(58 \%$ or $15 / 26)$, these differences were not significant.

Comparison between patients with oxacillin-sensitive and oxacillin-resistant Staphylococcus aureus $\underline{\text { bacteremia }}$

Oxacillin susceptibility was observed in 41\% (67/ 163 ) of the total cases studied. In hospital-acquired infections, oxacillin-resistant $S$. aureus was responsible for $64.3 \%(90 / 140)$ of the cases $(R R=2.46$ and $\left.\mathrm{CI}_{95 \%}=1.23-4.96\right)$. No cases of oxacillin resistance were observed in community-acquired infections (9/9; $\mathrm{p}=0.0002$ ). In cases of indeterminate origin, oxacillinsusceptible $S$. aureus was responsible for $57 \%$ (8/14). Bacterial susceptibility differed according to the patient's age. Susceptibility was lower for those over 60 years of age $\left(\mathrm{p}=0.018 ; \mathrm{CI}_{95 \%}=1.002-1.029\right)$, even when children under one year of age were excluded from the analysis. A greater incidence of resistant $S$. aureus was also observed among female patients $(\mathrm{RR}=1.26 ; \mathrm{CI}=0.98$ 1.63). Women, however, used a central catheter more than men (63\% of women, compared with $42 \%$ of men). As regards the hospital wards, oxacillin-susceptible $S$. aureus was predominant in the nursery $(\mathrm{p}=0.056)$ and oxacillin-resistant $S$. aureus was predominant in the intensive care units ( $\mathrm{p}=0.009)$.
Amongst the risk and comorbidity factors, univariate analysis showed the following association with oxacillinresistant $S$. aureus bacteremia: use of corticoids $\left(\mathrm{RR}=1.38 ; \mathrm{CI}_{95 \%}=1.08-1.77\right)$, previous surgery $\left(\mathrm{RR}=1.47 ; \mathrm{CI}_{95 \%}=1.15-1.87\right)$, mechanical ventilation $\left(\mathrm{RR}=1.48 ; \mathrm{CI}_{95 \%}=1.17-1.87\right)$, bladder probe $\left(\mathrm{RR}=1.55 ; \mathrm{CI}_{95 \%}=1.22-1.96\right)$, and presence of a central vascular catheter $\left(\mathrm{RR}=1.8 ; \mathrm{CI}_{95 \%}=1.36-2.39\right)$.

Univariate analysis showed a greater incidence of oxacillin-susceptible $S$. aureus phlebitis $(\mathrm{RR}=8.60$ and $\mathrm{CI}_{95 \%}=1.06-69.78$ ). Multiple logistical regression analysis, however, showed these data to be distorted by the shorter hospitalization time in cases of phlebitis (median=20 days) compared with the other cases (median $=35$ days). Bacteremia without a known focus was associated with susceptibility to oxacillin $(R R=2.24$ and $\mathrm{CI}_{95 \%}=1.30-3.86$ ), whereas central-catheter-related infection was associated with resistance $(\mathrm{RR}=3.91$ and $\left.\mathrm{CI}_{95 \%}=1.54-9.60\right)$. However, regression analysis showed this difference between primary bacteremias to be distorted by the longer hospitalization time in cases of central catheter-related infection (median $=33$ days in cases of bacteremia without a focus; median $=39$ days in cases of catheter-related infection).

A significant association was observed between oxacillin resistance and the use of antibiotics before the bacteremia. As regards the number of antibiotics, an association was observed with increasing resistance to oxacillin for each additional antibiotic in previous therapy $(\mathrm{p}<0.001$ and $\mathrm{CI}_{95 \%}=2.04-4.23$ ). As regards the length of time that antibiotics were previously used, an increase in resistance tooxacillin was observed for each additional day of antibiotic use $\left(\mathrm{p}<0.001\right.$ and $\left.\mathrm{CI}_{95 \%}=1.06-1.16\right)$. An average of 0.64 different antibiotics were used in the cases of susceptible $S$. aureus bacteremia (ranging from 0 to 6 ; median=0), and an average of 2.43 were used (ranging from 0 to 7 ; median=2) in the resistant $S$. aureus bacteremia cases.

Resistance to oxacillin in hospital-acquired infections was greatest in cases where duration of hospitalization exceeded seven days $(\mathrm{p}=0.017, \mathrm{RR}=2.67$ and $\mathrm{CI}_{95 \%}=1.8-8.91$ ).

Those variables that showed a significant association with the bacterial susceptibility profile in the univariate analysis are listed in Table 1. 
Table 1. Association between clinical and epidemiological variables and the profile of Staphylococcus aureus susceptibility to oxacillin in univariate analysis

\begin{tabular}{|c|c|c|c|}
\hline & Susceptible & Resistant & Statistical information \\
\hline \multicolumn{4}{|l|}{ Sex } \\
\hline Female & $22(33 \%)$ & $45(67 \%)$ & $\mathrm{RR}=1.26 ; \mathrm{CI}_{95 \%}=0.98-1.63$ \\
\hline \multicolumn{4}{|r|}{$95 \%$} \\
\hline$>60$ years & $22(33 \%)$ & $45(67 \%)$ & $\mathrm{RR}=1.2 ; \mathrm{CI}_{95 \%}=0.94-1.55$ \\
\hline \multicolumn{4}{|l|}{ Previous use of antibiotics } \\
\hline Number of antibiotics (per antibiotic) & - & - & $\mathrm{p}<0.001 ; \mathrm{CI}_{95 \%}=2.04-4.23$ \\
\hline Length of use (per day) & - & - & $\mathrm{p}<0.001 ; \mathrm{CI}_{95 \%}=1.06-1.16$ \\
\hline \multicolumn{4}{|l|}{ Origin } \\
\hline Community & $9(100 \%)$ & - & $\mathrm{RR}=0.0 ; \mathrm{CI}_{95 \%}=0.0-0.37$ \\
\hline Hospital & $50(36 \%)$ & $90(64 \%)$ & $\mathrm{RR}=2.46 ; \mathrm{CI}_{95 \%}=1.23-4.96$ \\
\hline \multicolumn{4}{|l|}{ Hospital ward } \\
\hline Intensive Care & $16(28 \%)$ & $42(72 \%)$ & $\mathrm{RR}=1.41 ; \mathrm{CI}_{95 \%}=1.1-1.8$ \\
\hline Nursery & $7(70 \%)$ & $3(30 \%)$ & $\mathrm{RR}=0.49 ; \mathrm{CI}_{95 \%}=0.19-1.28$ \\
\hline \multicolumn{4}{|l|}{ Risk factors } \\
\hline Corticoids & $9(25 \%)$ & $27(75 \%)$ & $\mathrm{RR}=1.38 ; \mathrm{CI}_{95 \%}=1.08-1.77$ \\
\hline Surgery & $15(26 \%)$ & $43(74 \%)$ & $\mathrm{RR}=1.47 ; \mathrm{CI}_{95 \%}=1.15-1.87$ \\
\hline Mechanical ventilation & $6(20 \%)$ & $24(80 \%)$ & $\mathrm{RR}=1.48 ; \mathrm{CI}_{95 \%}=1.17-1.87$ \\
\hline Bladder probe & $12(23 \%)$ & $41(77 \%)$ & $\mathrm{RR}=1.55 ; \mathrm{CI}_{95 \%}=1.22-1.96$ \\
\hline Central catheter & $20(24 \%)$ & $62(76 \%)$ & $\mathrm{RR}=1.80 ; \mathrm{CI}_{95 \%}=1.36-2.39$ \\
\hline \multicolumn{4}{|l|}{ Focus } \\
\hline Catheter with bacteremia & $5(15 \%)$ & $28(85 \%)$ & $\mathrm{RR}=3.91 ; \mathrm{CI}_{95 \%}=1.54-9.60$ \\
\hline Unknown focus & $25(61 \%)$ & $16(39 \%)$ & $\mathrm{RR}=2.24 ; \mathrm{CI}_{95 \%}=1.30-3.86$ \\
\hline Phlebitis & $6(86 \%)$ & $1(14 \%)$ & $\mathrm{RR}=8.6 ; \mathrm{CI}_{95 \%}=1.06-69.78$ \\
\hline Total cases & $67(41 \%)$ & $96(59 \%)$ & \\
\hline
\end{tabular}

Table 2. Association between clinical and epidemiological variables and the profile of Staphylococcus aureus susceptibility to oxacillin in multiple logistical regression analysis

\begin{tabular}{lccc}
\hline & RR & $\mathbf{p}$ & $\mathbf{C I}_{\mathbf{9 5 \%}}$ \\
\hline Age $>$ 60 years & 1.02 & 0.004 & $1.00-1.05$ \\
Corticoids & 3.22 & 0.033 & $1.1-9.42$ \\
Central catheter & 2.48 & 0.034 & $1.07-5.78$ \\
$\begin{array}{l}\text { Previous use of antibiotics } \\
\text { (for each antibiotic) }\end{array}$ & 3.02 & $<0.001$ & $2.03-4.51$
\end{tabular}

$\mathrm{RR}=$ relative risk

$\mathrm{CI}=$ confidence interval. 
It was found by multiple logistical regression analysis, for which the initial model included those variables that showed an association with resistance to oxacillin (sex, age, length of hospitalization, diabetes, use of corticoids, central catheter, bladder probe, mechanical ventilation, number of previous antibiotics, stay in Intensive Care, surgery carried out), that the model which best fits resistance to oxacillin in this study includes four variables: age greater than 60 years ( $p=0.004$ and $R R=1.02)$; use of catheter $(p=0.034$ and $R R=2.45)$; use of corticoids ( $p=0.033$ and $\mathrm{RR}=3.22$ ); and a greater number of antibiotics used before the bacteremia $(\mathrm{p}<0.001$ and $R R=3.02)$. The set of variables that showed the most significant association with the bacterial susceptibility profile in the multiple logistical regression analysis is shown in Table 2.

There was no significant relationship between mortality and resistance to oxacillin, even when deaths attributable to the bacteremia were analyzed. The association with death in the cases of oxacillinsusceptible $S$. aureus bacteremia was $40 \%$ (20/67), falling to $27 \%$ (18/67) when deaths up to 15 days after blood culture were selected. The association with death in the cases of oxacillin-resistant $S$. aureus bacteremia was $45 \%$ (43/96), falling to $33 \%$ (32/96) for deaths up to 15 days after blood culture.

\section{Discussion}

According to our results, the staphylococcal bacteremias seen in the IC-HC and InCor are predominantly hospital-acquired $(85.9 \%)$ and due to oxacillin-resistant $S$. aureus $(58.9 \%)$ There was a high mortality associated with staphylococcal bacteremia $(42.9 \%)$, as described in earlier studies [14,18]. Our study did not show any association between mortality and resistance to oxacillin. The presence of comorbidity factors may have contributed to the high rates of mortality. This is suggested by the strong presence of such factors amongst the patients, as well as by the large proportion of patients hospitalized in intensive care (36.5\% of the cases).
Although data can be found in the literature indicating an increasing incidence of community-acquired oxacillin-resistant $S$. aureus infections, it can be seen that most studies do not clearly show the criteria used to define community-acquired infection, which is frequently taken as an infection detected by culture within 48 or 72 hours of admission to hospital [2,5]. Based on the criteria adopted in our study, and in view of the greater accuracy of the data resulting from the prospective nature of the study, we did not detect any cases of community-acquired resistant $S$. aureus.

Infections by $S$. aureus strains that are resistant to oxacillin, but susceptible to clindamycin or sulphametoxazol-trimethoprim, have been described recently, both in the U.S. and in other parts of the world, and are associated with a genetic resistance mechanism. Many of these infections have been acquired in the community and are mainly associated with infections of the soft tissues [19,20].

The multiple regression analysis of the correlation between oxacillin-resistant $S$. aureus bacteremia and sex, age, length of hospitalization, diabetes, use of corticoids, central catheter, bladder probe, mechanical ventilation, number of previous antibiotics, stay in Intensive Care, and surgery carried out (which showed a significant association in univariate analysis) was deemed worthwhile. It was found by multiple regression analysis that the model which best fits resistance to oxacillin in this study includes four variables: age greater than 60 years $(\mathrm{p}=0.004)$; use of catheter $(p=0.034)$; use of corticoids $(p=0.033)$; and a greater number of antibiotics used before the bacteremia ( $p<0.001)$. Patients submitted to surgery and those who had been hospitalized for long periods showed a predominance of oxacillin-resistant $S$. aureus bacteremia as a result of the greater use of antibiotics in these cases.

The association that we found between resistance to oxacillin and central catheter as a focus suggests that this device is indeed one of the main factors, if not the most important factor, predisposing to infection of the bloodstream in our setting, particularly as it was observed that the catheter was the second-mostfrequently detected focus ( $20.2 \%$ of cases), second 
only to primary bacteremia without a known focus (25.2\%). Particular attention should therefore be paid to correct use of the central vascular catheter in hospital infection control. It is worth noting that bacteremia without a known focus and bacteremic catheter-related infection are standardized as the same type of focus (primary bacteremia). However, our results suggest that they may be considered different foci for the purposes of hospital infection control, as there was a clear difference between these two categories, both in terms of susceptibility to oxacillin $(61 \%$ in bacteremias without focus and $15 \%$ in those associated with a catheter) and in terms of length of hospitalization (medians equal to 33 and 39 days, respectively).

In spite of the limited sample size, a significant relationship between phlebitis and oxacillin-susceptible S. aureus was observed, which can be explained by the short stay in these cases. This information could be useful for therapeutic guidance.

\section{References}

1. Cookson B., Peters B., Webster M., et al. Staff carriage of epidemic methicillin-resistant Staphylococcus aureus. J Clin Microbiol 1989;27(7):1471-76.

2. Moreno F., Crisp C., Jorgensen J.H., Patterson J.E. Methicillin-resistant Staphylococcus aureus as a community organism. Clin Infect Dis 1995;21:1308-12.

3. Steinberg J.P., Clark C.C., Hackman B.O. Nosocomial and community-acquired Staphylococcus aureus bacteremias from 1980 to 1993: impact of intravascular devices and methicillin resistance. Clin Infect Dis 1996;23:255-9.

4. Mylotte J.M., McDermott C., Spooner A. Prospective study of 114 consecutive episodes of Staphylococcus aureus bacteremia. Rev Infect Dis 1987;9(5):891-907.

5. Conterno L.O., Wey S.B., Castelo A. Staphylococcus aureus bacteremia: comparison of two periods and a predictive model of mortality. Bras $\mathbf{J}$ Infect Dis 2002;6(6):288-97.

6. Smith T.L., Pearson M.L., Wilcox K.R., et al. Emergence of vancomycin resistance in Staphylococcus aureus. N Engl J Med 1999;340(7):493-501.

7. Sieradzki K., Roberts R.B., Haber S.W., Tomasz A. The development of vancomycin resistance in a patient with methicillin-resistant Staphylococcus aureus infection. N Engl J Med 1999;340(7):517-23.
8. Oliveira G.A., Dell'Aquila A.M., Masiero R.L., et al. Isolation in Brazil of nosocomial Staphylococcus aureus with reduced susceptibility to vancomycin. Infect Control Hosp Epidemiol 2001;22(7):443-8.

9. Fridkin S.K., Hageman J., McDougal L.K., et al. Epidemiological and microbiological characterization of infections caused by Staphylococcus aureus with reduced sensibility to vancomycin, United States, 19972001. Clin Infect Dis 2003;36(4):429-39.

10. CDC - Centers for Disease Control and Prevention. Vancomycin-resistant Staphylococcus aureus Pennsylvania, 2002. MMWR Morb Mortal Wkly Rep 2002;51(40):902.

11. Small P.M., Chambers H.F. Vancomycin for Staphylococcus aureus endocarditis in intravenous drug users. Antimicrob Agents Chemoter 1990;34(6):1227-31.

12. Romero-Vivas J., Rubio M., Fernandez C., Picazo J.J. Mortality associated with nosocomial bacteremia due to methicillin-resistant Staphylococcus aureus. Clin Infect Dis 1995;21:1417-23.

13. Harbarth S., Rutschmann O., Sudre P., Pittet D. Impact of methicillin resistance on the outcome of patients with bacteremia caused by Staphylococcus aureus. Arch Intern Med 1998; 158:182-9.

14. Cosgrove S.E., Sakoulas G., Perencevich E.N., et al. Comparison of mortality associated with methicillinresistant and methicillin-susceptible Staphylococcus aureus bacteremia: a meta-analysis. Clin Infect Dis 2003;36(1):53-9.

15. Moreira M., Medeiros E.A., Pignatari A.C., et al. Effect of nosocomial bacteremia caused by oxacillin-resistant Staphylococcus aureus on mortality and length of hospitalization. Rev Assoc Med Bras 1998;44(4):2638.

16. NCCLS - National Committee for Clinical Laboratory Standards. Performance Standards for Antimicrobial Susceptibility Testing (vol 19, $\left.\mathrm{n}^{\circ} 1\right) \mathbf{1 9 9 9 .}$

17. CDC - Centers for Disease Control and Prevention. NNIS Manual - National Nosocomial Infectious Surveillance System. 1992, XIII(1)-XIII(59).

18. Conterno L.O., Wey S.B., Castelo A. Risk factors for mortality in Staphylococcus aureus bacteremia. Infect Control Hosp Epidemiol 1998; 19:32-7.

19. CDC. Four pediatric deaths from community-Acquired methicillin-resistant Staphylococcus aureus Minnesota and North Dakota, 1997-1999. MMWR 1999;48:707-10.

20. Collignon P., Gosbell I., Vickery A., et al. Communityacquired methicillin-resistant Staphylococcus aureus in Australia. Lancet 1998;352:145-6. 